\title{
Importância das práticas alimentares no primeiro ano de vida na prevenção da deficiência de ferro'
}

\author{
Importance of food practices \\ during the first year of life to \\ prevent iron deficiency
}

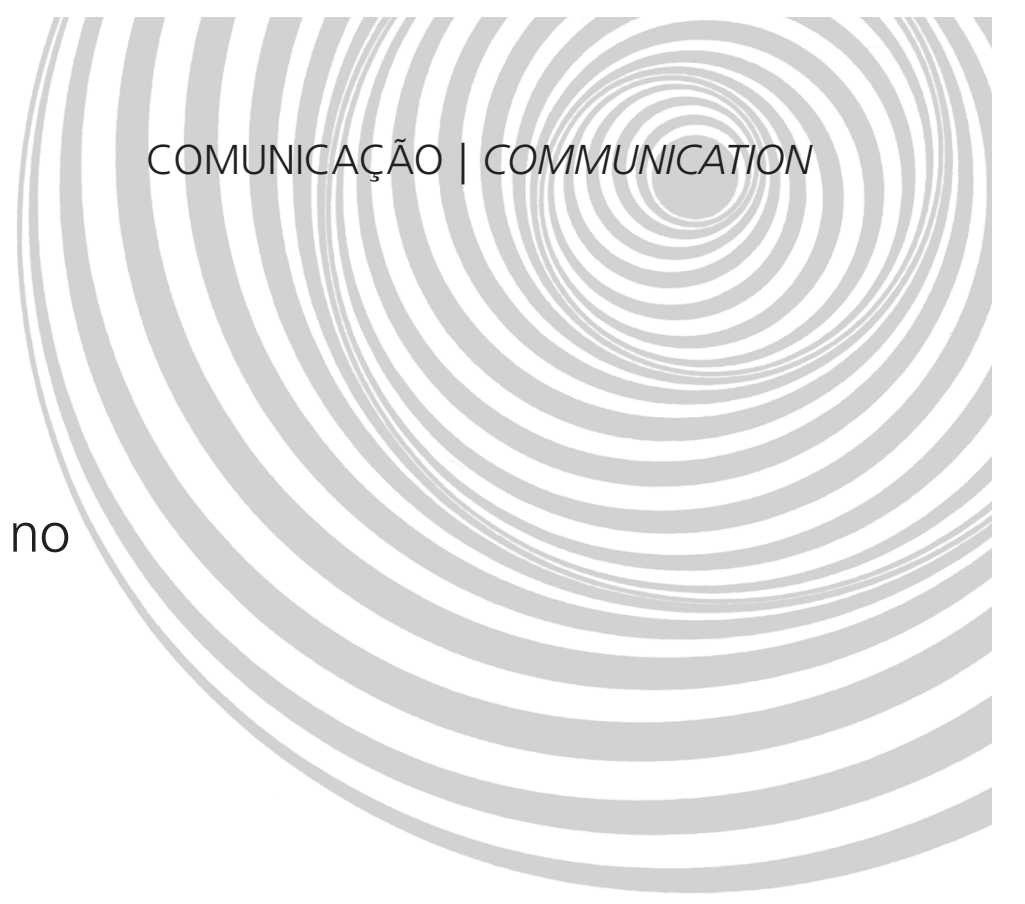

Gisele Ane BORTOLINI²

Márcia Regina VITOLO²

RE S U M O

A reconhecida relevância da anemia por deficiência de ferro, em termos de saúde pública, decorre não apenas da magnitude de sua ocorrência, mas, principalmente, dos efeitos deletérios que ocasiona à saúde da criança. Com o objetivo de investigar as práticas alimentares no primeiro ano de vida e sua associação com a deficiência de ferro, realizou-se revisão da literatura científica nacional e internacional sobre a questão, selecionando os artigos mais relevantes. Crianças que nascem a termo e com peso adequado, ao receberem o leite materno de forma exclusiva suprem suas necessidades de ferro, sendo desnecessário qualquer complemento nos primeiros seis meses de vida. Próximo aos seis meses de idade ocorre gradualmente o esgotamento das reservas de ferro e a alimentação complementar passa a ter papel predominante no atendimento às necessidades desse nutriente. O papel do aleitamento materno na ocorrência da deficiência de ferro é ainda controverso e parece depender do país, região e tipo de leite utilizado em substituição ao leite materno. Na impossibilidade da continuidade do aleitamento materno, a substituição deste por leite de vaca aumenta o risco de a criança apresentar deficiência de ferro. Práticas complementares que comprovadamente contribuem com a prevenção da deficiência de ferro são: alimentação complementar com alta biodisponibilidade de ferro, alimentos fortificados e suplemento de ferro em doses profiláticas. Dieta com alta biodisponibilidade de ferro é aquela que contém os alimentos básicos da família, desde que saudáveis, com a presença de carne, vitamina A e vitamina C. A alimentação no primeiro ano de vida tem papel importante na prevenção da anemia por deficiência de ferro e, para sua adequada implementação, é necessário que suas diretrizes sejam adotadas como rotina nas unidades básicas de saúde.

Termos de indexação: Alimentação. Anemia. Criança. Deficiência de ferro.

\footnotetext{
1 Artigo elaborado a partir da dissertação de G.A. BORTOLINI, intitulada "Anemia, deficiência de ferro e anemia por deficiência de ferro em crianças aos 12 meses e aos 4 anos de idade". Universidade Federal de Ciências da Saúde de Porto Alegre; 2008.

2 Universidade Federal de Ciências da Saúde de Porto Alegre, Programa de Pós-Graduação em Ciências da Saúde. R. Sarmento Leite, 245, 90050-170, Porto Alegre, RS, Brasil. Correspondência para/Correspondence to: G.A. BORTOLINI. E-mail: <giselebortolini@hotmail.com>.
} 
1052 | G.A. BORTOLINI \& M.R. VITOLO

\section{A B S T R A C T}

The well-known relevance of iron deficiency anemia for public health derives not only from its prevalence, but mainly from its harmful effects on child health. In order to investigate feeding practices in the first year of life and their association with iron deficiency, domestic and international scientific articles were reviewed and the most relevant articles were selected. Children born at term and with appropriate birth weight get all the iron they need from exclusive breastfeeding, with complementary foods of any kind being unnecessary in the first six months of life. At about six months of life, the iron reserves are gradually depleted and complementary foods take on a predominant role in meeting the iron requirement. The role of breastfeeding in the occurrence of iron deficiency is still debated, and appears to depend on the country, region and type of milk used to replace breast milk. The replacement of breast milk with cow's milk increases the risk of the child developing iron deficiency. Complementary practices that have been proven to contribute to the prevention of iron deficiency are complementary foods high in bioavailable iron, enriched foods and iron supplementation in prophylactic doses. A diet high in iron bioavailability is that which contains the staple foods of the family, as long as they are healthy, with the presence of meat, vitamin A and vitamin C. The foods consumed in the first year of life have an important role in the prevention of iron deficiency anemia, and their correct implementation depends on guidelines that should be adopted as standard practice by primary healthcare units.

Indexing terms: Feeding. Anemia. Child. Iron deficiency.

\section{N T R O D U ÇÃ O}

A Organização Mundial da Saúde (OMS), em recente publicação, apresentou uma análise global da prevalência de anemia no mundo, no período de 1993 a 2005. E estimou que, em termos globais, a anemia afeta 1,62 bilhões de pessoas (IC95\%; 1,50 - 1,74 bilhões). As crianças em idade pré-escolar são as mais afetadas, com prevalência de 47,4\% (293 milhões) ${ }^{1}$. Também estima que a prevalência de deficiência de ferro seja 2,5 vezes maior que a prevalência de anemia².

No Brasil, a Pesquisa Nacional de Demografia e Saúde de 2006³ , avaliou, pela primeira vez em nível nacional, a prevalência de anemia em crianças e observou que $20,9 \%$ das crianças de zero a 59 meses apresentam anemia, ou seja, aproximadamente três milhões de crianças apresentam anemia. As maiores prevalências foram observadas no Nordeste (25,5\%), Sudeste (22,6\%) e Sul (21,5\%). A Região Norte (10,4\%) e a Região Centro-Oeste $(11,0 \%)$ apresentaram as prevalências mais baixas. A ocorrência de anemia em crianças tem sido explorada por diversos estudos no Brasil nos últimos vinte anos. Um estudo de revisão realizado no país sumariza os resultados de 53 estudos realizados no período de 1996 a 2007, nos mais diversos âmbitos e mostrou que os dados medianos para a prevalência de anemia nesse mesmo grupo etário foi de 53,0\%, sendo que as maiores prevalências são observadas em crianças menores de 24 meses $^{4}$.

A reconhecida relevância da anemia por deficiência de ferro, em termos de saúde pública, não decorre apenas da magnitude de sua ocorrência, mas, principalmente, dos efeitos deletérios que ocasiona à saúde da criança, tais como repercussões negativas no desenvolvimento psicomotor e cognitivo, diminuição na capacidade de aprendizagem e comprometimento da imunidade celular com menor resistência às infecções ${ }^{5,6}$. Esses efeitos podem persistir mesmo após suplementação com ferro ${ }^{7}$. Evidência recente mostrou que duzentos milhões de crianças menores de cinco anos, residentes em países em desenvolvimento, não atingem seu potencial de desenvolvimento ${ }^{8}$. Na mesma série de trabalhos, Walker et al. ${ }^{9}$ identificaram quatro fatores de riscos prioritários para intervenções em nível de saúde pública mundial: baixa estatura, estimulação cognitiva, deficiência de iodo e anemia por deficiência de ferro, que ocorrem isoladamente ou, na maioria das vezes, concomitantemente, agravando a situação. A deficiência de ferro está entre os dez principais fatores de risco, indicados pela Organização Mundial da Saúde, que contribuem para a diminuição 
da qualidade e expectativa de vida no mundo ${ }^{10}$. A deficiência de ferro tem custo econômico alto somado à sobrecarga ao sistema de saúde, pois diminui a capacidade de produtividade em adultos $^{11}$. Ross \& Horton ${ }^{12}$ desenvolveram um algoritmo para estimar as perdas econômicas para os países, devido ao atraso no desenvolvimento cognitivo de crianças, a menor produtividade em adultos e os partos prematuros. Os autores sugerem que a perda é de US\$4,00 per capita/ano devido à produtividade reduzida em função da deficiência de ferro. Assim, o presente artigo tem por objetivo destacar a importância das práticas alimentares no primeiro ano de vida na prevenção da deficiência de ferro, com enfoque nas recomendações de ferro para crianças, aleitamento materno, alimentação complementar, fortificação de alimentos, suplementação com ferro e educação nutricional.

Foram utilizados para a revisão artigos sobre anemia e sobre alimentação complementar publicados em inglês, português e espanhol. As bases de dados utilizadas para a pesquisa foram MedLine, SciELO, PubMed, Bireme, Portal da Capes e publicações de organismos governamentais e não-governamentais.

\section{Práticas alimentares saudáveis com foco na prevenção da deficiência de ferro}

As reservas de ferro da criança, que recebe com exclusividade o leite materno, do nascimento aos seis meses de idade, suprem as necessidades fisiológicas da criança. Dessa forma, durante esse período não há necessidade de qualquer forma de complementação e nem introdução de alimentos sólidos ${ }^{1,2}$. Esse fato se deve à biodisponibilidade elevada do ferro no leite humano, sendo cerca de $50 \%$ de seu ferro absorvido, o que compensa a sua baixa concentração $(0,5-1 \mathrm{mg}$ de ferro/litro). Entretanto, essa biodisponibilidade pode diminuir até $80 \%$ quando outros alimentos passam a ser ingeridos pelo lactente. A quantidade de ferro presente no leite de vaca é de $0,6 \mathrm{mg} / \mathrm{L}$ e a absorção é de $10 \%{ }^{13}$. Próximo aos seis meses de idade, ocorre gradualmente o esgotamento das reservas de ferro e a alimentação passa a ter papel predominante no atendimento às necessidades desse nutriente ${ }^{14,15}$. É necessário que o consumo de ferro seja adequado à demanda requerida para essa fase etária.

Os principais fatores determinantes dos requerimentos de ferro durante a infância são as reservas de ferro ao nascer, as necessidades para o crescimento e a necessidade para repor as perdas. O requerimento basal de ferro é a quantidade necessária para manter as funções orgânicas, para reparar perdas normais e prover o crescimento corporal do indivíduo, regenerando e mantendo o estoque de reservas do nutriente, como uma segurança contra um futuro aumento das necessidades ou diminuição da ingestão de ferro ${ }^{16}$.

A recomendação de ferro é de $0,27 \mathrm{mg} / \mathrm{dia}$ do nascimento aos seis meses, $11 \mathrm{mg} /$ dia dos sete aos doze meses e de $7 \mathrm{mg} /$ dia do primeiro ao terceiro ano de vida. É importante observar que para todos os nutrientes disponíveis (vitaminas e minerais) para as duas faixas menores de um ano (0 - 6 e 7 a 12) os únicos que estão determinados como Recommended Dietary Allowance (RDA) no período de sete a doze meses são o ferro e o zinco. Os outros nutrientes têm como referência Adequate Intake (Al) que é uma sugestão de recomendação, pois é baseada no aporte de leite materno por esse ser fonte relevante de todos os micronutrientes com exceção do ferro e zinco, os quais, a partir dos seis meses, devem ter boas fontes na alimentação complementar ${ }^{16}$.

\section{Aleitamento materno}

Com o acúmulo de evidências sobre os benefícios do aleitamento materno exclusivo, a Organização Mundial da Saúde recomenda que as crianças sejam amamentadas exclusivamente por seis meses e que a criança receba a alimentação complementar e o leite materno dos seis meses até os 24 meses de idade. A OMS também evidência que são necessários futuros estudos 
para avaliar o risco da deficiência de micronutrientes, especialmente em crianças susceptíveis (que moram em áreas com elevada prevalência de deficiência de ferro, zinco e vitamina $A)^{17}$. No Brasil, nas últimas décadas, vem ocorrendo aumento na duração do aleitamento materno. No entanto, expressiva parcela dos lactentes é desmamada precocemente. A II Pesquisa Nacional de Aleitamento Materno, realizada no ano de 2008, mostrou que a duração mediana do aleitamento materno exclusivo foi de 54, 1 dias (1,8 meses) e a duração mediana do Aleitamento Materno foi de 341,6 dias (11,2 meses) no conjunto das capitais brasileiras e Distrito Federal ${ }^{18}$. 0 aleitamento materno exclusivo até os seis meses de idade e a manutenção do leite materno a partir dessa idade, juntamente com a alimentação complementar, estão associados a menor prevalência de anemia ${ }^{19}$, porém, o papel do aleitamento na anemia, deficiência de ferro e anemia por deficiência de ferro permanece controverso.

Estudo realizado em unidade básica de saúde, em Salvador (BA), por Assis et al. ${ }^{20}$, mostrou que a concentração média da hemoglobina das crianças que receberam leite materno de forma exclusiva por seis meses foi significativamente maior do que a apresentada pelas crianças submetidas às demais práticas alimentares. Em outro estudo, Szarfarc et al. ${ }^{21}$ observaram que as crianças da cidade de Santo André (SP) que recebiam o leite materno como única fonte de leite tiveram menor prevalência de anemia. Duarte et al. ${ }^{22}$, em estudo transversal de base populacional realizado na cidade de Itupeva (São Paulo), observaram que os resultados encontrados sugeriram associação entre presença de aleitamento materno e maiores níveis de hemoglobina somente entre as crianças com idade inferior a seis meses. Outros estudos no Brasil não observaram a proteção do aleitamento materno na ocorrência de anemia em crianças $^{23-26}$. Estudo realizado nos Estados Unidos mostrou que crianças amamentadas exclusivamente até seis meses apresentam maior risco de desenvolver deficiência de ferro quando comparadas às crianças que mamaram por menor tem$\mathrm{po}^{27}$. O papel do aleitamento materno parece depender do país, região e tipo de leite utilizado em substituição ao leite materno ${ }^{28-31}$, pois em países em desenvolvimento o substituto de leite materno é o leite de vaca, assim, os estudos que comparam o uso de leite materno em relação ao leite de vaca, mostram os benefícios do leite materno, porém, estudos realizados em países desenvolvidos, que fazem uso da fórmula infantil modificada como substituto, e comparam crianças que recebem leite materno com crianças que recebem fórmula infantil, mostram risco para anemia quando a criança recebe leite materno.

\section{Aleitamento artificial}

A introdução precoce de leite de vaca causa impacto negativo nos estoques de ferro das crianças por causa da baixa quantidade de ferro que esse contém (2,6mg de Fe para 1000kcal do alimento) e pela baixa biodisponibilidade, além de provocar micro-enterorragias pela imaturidade do trato gastrointestinal, provocando perdas sanguíneas $^{13}$. Estudo realizado por Souza et al. ${ }^{32}$ avaliou a prática alimentar de crianças de um ano de idade, atendidas em centros de saúde-escola de São Paulo. Aos três meses, cerca de $60 \%$ das crianças já estavam em processo de desmame e após seis meses de idade, a dieta continuava sendo predominantemente láctea. Estudo realizado por Levy-Costa \& Monteiro ${ }^{33}$, em São Paulo, com objetivo de estudar a influência do consumo de leite de vaca sobre a concentração de hemoglobina e o risco de anemia em crianças menores de cinco anos mostrou que a razão de chances para a anemia cresce em 50\% e 100\%, respectivamente, quando as crianças com consumo intermediário e alto de leite de vaca são comparadas às crianças com baixo consumo. Outro estudo realizado por Gunnarsson et al. ${ }^{34}$, realizado na Islândia, mostrou que o consumo de leite de vaca fluído está negativamente associado com os níveis de hemoglobina, 50\% das crianças que consumiam mais de $500 \mathrm{~mL} /$ dia de leite apresentaram deficiência de ferro, enquanto apenas $2 \%$ das crianças que consumiam menos do que essa quanti- 
dade apresentaram-se deficientes. Na impossibilidade da continuidade do aleitamento materno, a substituição deste por leite de vaca é fator de risco para deficiência de ferro e a substituição por fórmula infantil modificada é fator de proteção $14,15,34$.

\section{Alimentação complementar}

A II Pesquisa Nacional de Aleitamento materno, realizada no ano de 2008, avaliou pela primeira vez no Brasil a prática alimentar de crianças menores de um ano das capitais brasileiras e revelou resultados preocupantes. O consumo alimentar referente ao dia anterior da investigação mostrou que $70,0 \%$ das crianças de seis meses a nove meses consumiram frutas e $70,9 \%$ verduras/ legumes. No entanto, observa-se alta frequência do consumo de alimentos não saudáveis como café $(8,7 \%)$, refrigerante $(11,6 \%)$ e biscoitos/salgadinhos (71,7\%) em crianças de 9 a 12 meses $^{18}$.

A alimentação complementar deve ser oferecida a partir dos seis meses de idade da criança e o leite materno deve ser oferecido em complemento até dois anos ou mais. A partir dos seis meses as crianças devem receber uma papa de fruta no meio da manhã, uma papa salgada no almoço, uma de fruta no meio da tarde e leite materno em livre demanda. A partir de sete meses as crianças já podem receber a segunda papa salgada que deve ser oferecida no final da tarde. Todas as frutas devem se oferecidas para as crianças, não existem restrições, com preferências para as frutas regionais (Quadro 1). As papas salgadas, desde o início, já devem conter um alimento de cada grupo, inclusive carne que é a mais importante fonte de ferro (cereais ou tubérculos, leguminosas, verduras ou legumes ou frutas e as carnes ou ovo). A comida da família deve ser oferecida a partir dos dez meses, desde que não contenha muitos condimentos e sódio, e com um ano de idade a criança já pode receber as refeições básicas da família ${ }^{34}$.

Recomenda-se que todas as crianças recebam uma vez por semana fígado bovino, que é fonte de ferro heme. É importante o consumo de alimentos fonte de vitamina $C$, durante e após as papas e refeições de sal, pois a vitamina $C$ potencializa a absorção do ferro inorgânico presente nos alimentos. É importante a oferta de água, assim que outros alimentos forem introduzidos. Não se recomenda a oferta de sucos ou outras bebidas açucaradas ${ }^{35}$. A prática de oferecer bebidas açucaradas nos lanches e nos intervalos está associada à ocorrência de excesso de peso e cárie em crianças. Alimentos com alta concentração de açúcares e gorduras também não são recomendados antes de dois anos de idade, para a prevenção do excesso de peso, alergias e cárie. Considerando que os hábitos alimentares são formados nos primeiros anos de vida, esses alimentos não devem ser oferecidos para não induzir as crianças à preferência por esses alimentos não saudáveis ${ }^{35,36}$. No Brasil, o Ministério da Saúde sumariza as principais recomendações de ali-

Quadro 1. Esquema de introdução dos alimentos complementares.

\begin{tabular}{lll}
\hline Ao completar 6 meses & Ao completar 7 meses & Ao completar 10 meses \\
\hline Fruta & Fruta & Fruta \\
Papa salgada ${ }^{1}$ & Papa salgada & Refeição básica da família ${ }^{2}$ \\
Fruta & Fruta & Fruta \\
& Papa salgada & Refeição básica da família \\
\hline
\end{tabular}

1Papa salgada: embora e expressão "papa salgada" seja utilizada diversas vezes ao longo do texto, por ser conhecida e de fácil tradução para os pais na orientação da composição da dieta da criança e para diferenciá-la das papas de frutas, é importante salientar que o objetivo do uso do termo "salgada" não é adjetivar a expressão, induzindo ao entendimento de que a papa tenha muito sal ou que seja uma preparação com utilização de leite acrescido de temperos/sal. Expressões como "papa de vegetais com carne" ou outra que dê ideia de consistência (de papa) e variedade também podem ser empregadas como outras estratégias para uma boa comunicação em saúde; ${ }^{2}$ Refeição básica da família: com dez meses de idade a criança já pode receber os alimentos preparados para a família, desde que sem condimentos, sem muito sal ou temperos fortes. 
mentação complementar em uma publicação denominada "Dez Passos da Alimentação Saudável para Crianças Brasileiras Menores de Dois Anos" 35 .

\section{Biodisponibilidade do ferro}

É reconhecido que, mais importante do que suprir as necessidades, deve-se dar atenção à quantidade de ferro biodisponível presente nas refeições, o qual tem relação com os fatores estimulantes e inibidores de sua utilização numa mesma refeição ${ }^{37,38}$. Estudo realizado por Vitolo \& Bortolini ${ }^{37}$ mostrou que a biodisponibilidade do ferro dietético é o fator de maior importância na ocorrência de anemia ferropriva, 64,2\% das crianças que não apresentaram anemia possuíam dieta de alta biodisponibilidade em ferro e apenas 45,5\% das crianças com níveis de hemoglobina abaixo do parâmetro apresentaram esse tipo de dieta.

O ferro apresenta-se nos alimentos sob duas formas: heme e não heme. O ferro heme presente na hemoglobina e mioglobina das carnes e vísceras tem maior biodisponibilidade, não estando exposto a fatores inibidores, sendo que sua absorção é de $20-30 \%{ }^{38}$. Há evidência que o consumo de carne durante o período de desmame é fator importante na manutenção das reservas de ferro $15,37,39$.

O ferro não heme, contido no ovo, nos cereais, nas leguminosas (feijão) e nas hortaliças (beterraba), ao contrário do ferro animal, é absorvido de $2 \%$ a $10 \%$ pelo organismo. A absorção da forma não hemínica é fortemente influenciada por vários componentes da dieta, podendo ser inibida ou facilitada ${ }^{38,40}$. Três potentes facilitadores da absorção do ferro não heme são as carnes ${ }^{40}$, o ácido ascórbico ${ }^{41}$ e a vitamina $A^{42}$. Estudo realizado por Vitolo \& Bortolini ${ }^{37}$ mostrou que as crianças que não apresentaram anemia consumiram significativamente maior quantidade de vitamina C do que as que apresentaram, colocando em evidência o efeito benéfico desse micronutriente na prevenção da anemia, especialmente em estra- tos populacionais em que o consumo de carne é limitado devido às condições econômicas. Outros estudos também demonstraram a importância do consumo da vitamina $C$, como agente facilitador da absorção do ferro não heme e resultando em melhor estado nutricional de ferro ${ }^{15,41,43}$. Fitatos, taninos, cálcio e fosfatos, por outro lado, possuem efeito inibidor ${ }^{40,44}$. Para uma dieta de maior biodisponibilidade, deve-se aumentar o consumo de alimentos que contém os elementos facilitadores e diminuir o consumo dos que possuem fatores inibidores ${ }^{1,2,13,39,40}$.

As dietas podem ser classificadas tipicamente em três categorias de biodisponibilidade de ferro: baixa, intermediária e alta e a absorção média de ferro heme e não heme é aproximadamente 5, 10 e 15\%, respectivamente (em indivíduos com baixas reservas de ferro, mas com concentrações normais de hemoglobina) ${ }^{38}$. Dieta com baixa biodisponibilidade (5-10\%): é simples, em geral monótona, baseada em cereais, raízes e tubérculos com negligenciáveis quantidades de carne, peixe ou vitamina C. E contém predominantemente alimentos que inibem a absorção do ferro, como arroz, feijão, milho e farinha de trigo integral. Dieta com biodisponibilidade intermediária (11-18\%): é composta principalmente de cereais, raízes e tubérculos, mas inclui alguns alimentos de origem animal e/ou ácido ascórbico. A dieta com alta biodisponibilidade (>19\%) é diversificada e contém generosas quantidades de carne, aves, peixe e/ou alimentos ricos em ácido ascórbico.

\section{Educação nutricional}

A estratégia de educação nutricional que visa o consumo quantitativo e qualitativo adequado de alimentos fontes dos diversos nutrientes é uma alternativa que possui baixo custo e não produz efeitos indesejáveis. Por meio dela é possível aumentar o conhecimento da população sobre a deficiência de ferro e esclarecer quanto às dietas monótonas e pobres em ferro que se constituem uma das principais causas dessa deficiência. 
Ressalta-se, porém, que modificações nos hábitos alimentares não são rapidamente alcançadas, tornando a estratégia efetiva em longo prazo. Estudos de intervenção por meio de ações educativas voltadas para pais de crianças menores de 24 meses foram efetivos em prevenir a deficiência de ferro. Esses resultados confirmam que, a adesão às práticas alimentares corretas é importante para combater esse problema que apresenta alta prevalência nessa faixa etária ${ }^{45,46}$. Entretanto, para que haja resultados positivos suas ações devem garantir o consumo de alimentos ricos em ferro e estratégias dietéticas que aumentem a biodisponibilidade do ferro da alimentação, além de diminuir os fatores que a prejudicam.

A Organização Mundial da Saúde ${ }^{47}$ sumarizou recentemente em uma única publicação um curso de Aconselhamento em Alimentação Infantil, que foi traduzido no Brasil e aplicado em estudo de intervenção randomizado para avaliar a aplicabilidade do instrumento em capacitar profissionais de saúde. O estudo mostrou que para alguns itens do manual houve aumento de conhecimento por parte dos profissionais ${ }^{48}$, faltam pesquisas que avaliem se as orientações sobre alimentação complementar propostas pela OMS são efetivas na prevenção da deficiência de ferro. No Brasil, o Ministério da Saúde, por meio da Coordenação-Geral de Alimentação e Nutrição (CGAN) em parceria com a Rede Internacional em Defesa do Direito de Amamentar (IBFAN Brasil) propuseram a Estratégia Nacional para Alimentação Complementar Saudável (ENPACS) como um instrumento de fortalecimento às ações de apoio e promoção à alimentação complementar saudável no Sistema Único de Saúde. A ENPACS tem como objetivo incentivar a orientação da alimentação complementar como atividade de rotina nos serviços de saúde, contemplando a formação de hábitos alimentares saudáveis desde a infância, com a introdução da alimentação complementar em tempo oportuno e de qualidade, respeitando a identidade cultural e alimentar das diversas regiões brasileiras ${ }^{49}$.

\section{Fortificação de alimentos}

Uma das propostas para prevenção da deficiência de ferro é o estímulo ao consumo de alimentos fortificados, fortificação de alguns alimentos específicos, consumidos por alguns grupos populacionais ou fortificação em massa, como é o caso das farinhas de trigo e milho de alguns países, que é aceita, no âmbito de políticas públicas, como o melhor meio para combater carências específicas de nutrientes em longo prazo, apresentando como grande vantagem sua efetividade.

Os estudos sobre fortificação mostram resposta positiva, tanto em relação à aceitação do alimento fortificado, e na prevenção, como na recuperação dos níveis de hemoglobina nos grupos estudados ${ }^{50-52}$. As indústrias de alimentos têm utilizado o enriquecimento de seus produtos como um apelo comercial, focado para a criação de um atributo de qualidade adicional para incrementar a comercialização de seus produtos. Ainda não há dados no Brasil que avaliem o impacto do consumo desse alimentos, fortificados voluntariamente pela indústria, na prevalência de anemia. Em função do custo mais elevado de tais produtos, o impacto poderá não ser observado na população em geral.

No Brasil, desde 18 de junho de 2004, toda a farinha de trigo e milho comercializada no país é fortificada com ferro. A determinação é da Agência Nacional de Vigilância Sanitária (ANVISA), por Resolução $\operatorname{RDC} n^{\circ} 344^{53}$. O regulamento prevê que os fabricantes devem adicionar a cada 100 gramas de farinha no mínimo 4,2mg de ferro (30\% das IDR) e no mínimo 150 $\mu$ g de ácido fólico (70\% da IDR). A fortificação deixou de ser facultativa e passou a ser obrigatória. Essa medida objetivou aumentar a disponibilidade de alimentos ricos em ferro e ácido fólico para a população brasileira e, assim, contribuir para a redução da prevalência de anemia e defeitos do tubo neural no país. Todavia, essa medida torna-se questionável em relação aos lactentes, faixa etária de maior risco para anemia, devido ao fato desses alimentos não serem 
recomendados e consumidos regularmente em quantidades suficientes para atender às necessidades de ferro desse grupo em particular. Além disso, é provável que o baixo nível de ferro elementar $(40 \mathrm{mg} / \mathrm{kg})$ adicionado à farinha de trigo tenha pouco impacto no estado nutricional das crianças. Nenhum efeito da fortificação das farinhas foi observado nos níveis de hemoglobina das crianças menores de cinco anos, na cidade de Pelotas. Fato que, segundo os autores, pode ser parcialmente explicado pelo consumo insuficiente de farinhas e/ou pela baixa biodisponibilidade do ferro adicionado. $O$ estudo foi realizado entre maio e junho de 2004, anteriormente à obrigatoriedade de fortificação das farinhas e após 12 e 24 meses da implantação da medida $(2005$ e 2006)54. Além disso, no Brasil, ainda não existe um programa de monitoramento da fortificação obrigatória das farinhas.

Sherry et al. ${ }^{55}$, em estudo transversal utilizando dados do Center for Disease Control and Prevention's (CDC), de cinco Estados Americanos, observaram que a prevalência de anemia em crianças diminuiu em mais de $50 \%$ nas últimas duas décadas e foi atribuída às melhores condições de nutrição relacionadas ao consumo em larga escala de alimentos enriquecidos e possivelmente a melhor biodisponibilidade do ferro em alguns produtos. A prevalência de anemia (NHANES III - realizada entre 1988 - 1994) nos Estados Unidos foi de 3\% e 9\% nas crianças com idade de um a dois anos e menos de $1 \%$ e $3 \%$ nas crianças de três a cinco anos com anemia por deficiência de ferro e deficiência de ferro, respectivamente $^{56}$.

Outro país, exemplo, é o Chile em que a prevalência de anemia por deficiência de ferro é baixa em lactentes, pré-escolares, escolares, adolescentes, homens adultos e mulheres em idade fértil. Somente as mulheres grávidas ainda apresentam prevalência elevada. É provável que essa baixa prevalência se deva a fortificação das farinhas com ferro e vitaminas do complexo B, que acontece desde 1951 e ao Programa Nacional de Alimentación Complementaria (PNAC) que distribui leite para crianças desde 1952, sendo que, desde 1970, este é enriquecido com ferro. Através de estudos, a composição do produto foi sendo modificada e hoje o programa fornece para lactentes e gestantes leite em pó fortificado com ferro, zinco, cobre e ácido ascórbico ${ }^{57}$.

Na Europa, alguns países adotaram a política de distribuição de fórmulas infantis e cereais fortificados, que resultou na diminuição da prevalência de deficiência de ferro nas últimas décadas ${ }^{58}$. Cuba também adotou como uma das estratégias para combate da anemia ferropriva a fortificação de alimentos, sendo que as farinhas são enriquecidas com ferro e outras vitaminas e minerais desde 1999. As crianças menores de dois anos são prioridade de intervenção, sendo que mais de 95\% das crianças do país recebem, a preços subsidiados, uma papa de fruta enriquecida com ferro e vitamina C (desde 2001). Leite enriquecido com ferro é distribuído, também a preços subsidiados, para crianças menores de um ano desde 2005, e o programa tem cobertura de $98 \%{ }^{59}$. No Panamá distribui-se para as crianças, gratuitamente, através do Programa de Alimentación Complementaria (PAC), cereal enriquecido com vitaminas e minerais. Crianças em idade escolar recebem leite enriquecido com ferro desde 2006 e biscoitos que são oferecidos aos escolares do país também foram enriquecidos com ferro e outras vitaminas e minerais, com cobertura nas áreas de difícil acesso ${ }^{60}$.

Para crianças menores de um ano, a estratégia mais adequada parece ser a fortificação dos alimentos da criança em casa. A Bolívia é o primeiro país que documentou o uso da fortificação caseira, com intervenção em nível de saúde pública. No ano de 2005, os dados do país apontavam para prevalência de $70 \%$ de anemia em crianças de 6 a 24 meses. Para enfrentar tal problema, o país adotou a estratégia de distribuir sachês contendo ferro, vitamina $A$, vitamina $C$, ácido fólico e zinco para todas as crianças. O produto denomina-se "Chispitas" e cada criança recebe sessenta sachês por ano, para adicionar um sachês por dia em uma das refeições. A fortificação caseira 
teve início com a proposta dos "Sprinkles", no ano de 1996, por um grupo de pesquisa do The Hospital for Sick Children afiliado à Universidade de Toronto no Canadá e a estratégia foi denominada "home fortification". A eficácia biológica, biodisponibilidade, segurança e aceitabilidade foram testadas em diversos cenários, incluindo países como Bangladesh, Benin, Bolívia, China, Canadá, Ghana, Guiana, Haiti, Índia, Indonésia, Quirquistão, México, Mongólia, Paquistão, Vietnã ${ }^{61}$.

\section{Suplementação com ferro}

Na vigência de padrões alimentares que não incluem alimentos fortificados e/ou enriquecidos ou a prevalência de anemia em crianças menores de dois anos é severa (em torno de $40 \%$ ), a Organização Mundial da Saúde ${ }^{2}$ recomenda o uso universal de suplementos de ferro na dose de $2 \mathrm{mg} / \mathrm{kg}$ de peso ao dia, para todas as crianças de 6 a 23 meses de idade. A Sociedade Brasileira de Pediatria (SBP) recomenda a suplementação da dose $1 \mathrm{mg} / \mathrm{kg} /$ dia até dois anos de idade, para crianças que nasceram a termo e que não recebem fórmula infantil modificada ${ }^{62}$. Existem, atualmente, opiniões divergentes principalmente nos aspectos de periodicidade (e consequentemente sobre a dose administrada) da suplementação preventiva. A suplementação diária com sais de ferro é a forma de administração classicamente adotada. As críticas à suplementação preventiva têm como argumento a baixa adesão na prescrição dos profissionais de saúde e pelas mães na oferta do suplemento a seus filhos ${ }^{63}$.

Em termos de saúde pública, no Brasil, dispõe-se do Programa Nacional de Suplementação de ferro, que está em fase de implantação, o qual prevê a distribuição de suplementos de ferro para todos os municípios brasileiros, com o objetivo da suplementação universal de crianças de 6 a 18 meses com dose semanal de $25 \mathrm{mg}$ de ferro. Para melhorar a palatabilidade do sulfato ferroso e reduzir efeitos colaterais, foi desenvolvido um xarope de sulfato ferroso com gosto de fruta cítrica (laranja), na concentração de $25 \mathrm{mg}$ de ferro para $5 \mathrm{~mL}$ do produto ${ }^{64}$. A dosagem semanal recomendada pelo programa foi avaliada por um ensaio de campo randomizado com crianças de 6 a 12 meses de idade, na cidade do Rio de Janeiro, e teve por objetivo avaliar a efetividade da suplementação de ferro, em administração diária e semanal na prevenção de anemia. A intervenção foi realizada por 24 meses e ao final do estudo mostrou que a prevalência de anemia no grupo que recebeu suplementação com ferro em dose diária de $12,5 \mathrm{mg}$ foi de $50,6 \%$ e no grupo com suplementação semanal de $25 \mathrm{mg}$ foi de $60,5 \%$.

Outro estudo piloto realizado na cidade São Leopoldo (RS) mostrou que a adesão à dose semanal de ferro é baixa e que não resultou em menor prevalência de anemia. A prevalência de anemia, segundo o referencial adotado de hemoglobina $<11,0 \mathrm{~g} / \mathrm{dL}$, não diferiu entre os grupos, $48,2 \%$ no grupo intervenção (dose semanal de $25 \mathrm{mg}$ ) e $50,0 \%$ no grupo controle (dose prescrita segundo rotina da unidade de saúde). Na avaliação realizada no final do primeiro ano de vida, apenas $44,6 \%$ das crianças do grupo intervenção ainda estavam recebendo o suplemento de ferro. Os motivos citados pelas mães das crianças para ou não utilizara suplementação de ferro foram: doença da criança $(22,5 \%)$, intolerância digestiva $(45,3 \%)$ e a mãe não quis e/ou esqueceu $(32,2 \%)^{66}$.

Nos últimos 150 anos ou mais, o xarope de sulfato ferroso tem sido usado como a primeira estratégia para o controle da anemia por deficiência de ferro em crianças ${ }^{67}$. No entanto, a adesão ao uso de sulfato ferroso é frequentemente limitada pela combinação de alguns fatores: o gosto desagradável, o longo período de administração e alguns possíveis efeitos colaterais ${ }^{66,68,69}$.

\section{O N SI DER A ÇÃ O FIN A L}

Apesar das medidas individuais e populacionais adotadas no país, mantém-se a elevada prevalência de anemia, a qual incide em cerca de 
$40 \%$ das crianças menores de cinco anos há duas décadas. A anemia é determinada por diversos fatores, assim, várias ações são necessárias para sua prevenção. No entanto, a alimentação no primeiro ano de vida é um dos principais determinantes desse processo. $\mathrm{O}$ aleitamento materno e a alimentação complementar saudável têm papel importante na prevenção da deficiência de ferro quando associada a outras medidas e essas práticas podem ser bem sucedidas por meio de ações efetivas dos profissionais de saúde. Enfatiza-se a necessidade da incorporação de ações de aconselhamento sobre alimentação infantil integrando conteúdos, como práticas permanentes, às políticas de atenção à saúde da criança, ressaltando-se a necessidade da contínua atualização dos profissionais envolvidos.

\section{COLABORADORES}

G.A. BORTOLINI e M.R. VITOLO participaram de todas as fases do artigo.

\section{REFERÊ NCIAS}

1. World Health Organization. Nutritional anemia. sight and life press. Basel: WHO; 2007.

2. World Health Organization. Iron deficiency anaemia: assessment, prevention, and control. a guide for programme managers. Geneva: WHO; 2001.

3. Brasil. Ministério da Saúde. Pesquisa Nacional de Demografia e Saúde da Criança e da Mulher - PNDS 2006: dimensões do processo reprodutivo e da saúde da criança. Brasília: Ministério da Saúde; 2009.

4. Jordão RE, Bernardi JLD, Barros Filho A. Prevalência de anemia ferropriva no Brasil: uma revisão sistemática. Rev Paul Pediatr. 2009; 27(1):90-8.

5. Walter T, De Andraca I, Chadud P, Perales CG. Iron deficiency anemia: adverse effects on infant psychomotor development. Pediatrics. 1989; 84(1):7-17.

6. Grantham-McGregor S, Ani C. A review of studies on the effect of iron deficiency on cognitive development children. J Nutr. 2001; 131:S649-S68.

7. Lozoff B, Jimenez E, Smith JB. Double burden of iron deficiency in infancy and low socioeconomic status: a longitudinal analysis of cognitive test scores to age 19 years. Arch Pediatr Adolesc Med. 2006; 160(11):1108-13.

8. Grantham-McGregor S, Cheung YB, Cueto $S$, Glewwe P, Richter L, Strupp B, et al. Developmental potential in the first 5 years for children in developing countries. Lancet. 2007; 369(9555): 60-70.

9. Walker SP, Wachs TD, Gardner JM, Lozoff B, Wasserman GA, Pollitt E, et al. Child development: risk factors for adverse outcomes in developing countries. Lancet. 2007; 369(9556):145-57.

10. Ezzati M, Lopez AD, Rodgers A, Vander Hoorn S, Murray CJ, Comparative risk assessment collaborating group: selected major risk factors and global and regional burden of disease. Lancet. 2002; 360(9343):1347-60.

11. Haas JD, Brownlie TT. Iron deficiency and reduced work capacity: a critical review of the research to determine a casual relationship. Nutrition. 2001; 131(2S-2):676S-88S.

12. Ross R, Horton S. Economic consequences of iron deficiency. The Micronutrient Iniciative; 1998.

13. United Nations Children's Fund. Preventing iron deficiency in women and children. Boston: International Nutrition Foundation; 1999.

14. Male C, Persson LA, Freeman V, Guerra A, Van't Hof MA, Haschke F, et al. Prevalence of iron deficiency in 12-mo-old infants from 11 European áreas and influence of dietary factors on iron status (Euro-Growth Study). Acta Paediatrics. 2001; 90(5): 492-98.

15. Devincenzi MU, Colugnati FAB, Singulem DM. Factores de protección para la anemia ferropriva: estudio prospectivo en niños de bajo nivel socioeconómico. Arch Latinoam Nutr. 2004; 54(2):174-9.

16. Institute of Medicine. Dietary Reference intakes for vitamin A, vitamin K, arsenic, boron, chromium, copper, iodine, iron, manganese, molybdenum, nickel, silicon, vanadium and zinc. Washington (DC): The National Academy Press; 2001.

17. World Health Organization. Nutrient adequacy of exclusive breastfeeding for the term infant during the firts six months of life. Geneva: WHO; 2002.

18. Brasil. Ministério da Saúde. II pesquisa de prevalência de aleitamento materno nas capitais brasileiras e distrito federal. Brasília: Ministério da Saúde; 2009.

19. Dewey KG, Cohen RJ, Rivera LL, Brown KH. Effects of age of introduction of complementary foods on iron status of breast-fed infants in Honduras. Am J Clin Nutr. 1998; 67(5):878-84. 
20. Assis AM, Barreto ML, Gomes GS, Prado MS, Santos NS, Santos LM, et al. Childhood anemia prevalence and associated factors in Salvador. Cad Saúde Pública. 2004; 20(6):1633-41.

21. Szarfarc SC, Berg G, Santos AL, Souza SB, Monteiro CA. Prevenção de anemia no primeiro ano de vida em centros de saúde do município de Santo André, São Paulo. J Pediatr (Rio de Janeiro). 1996; 72(5): 329-34.

22. Duarte LS, Fujimori E, Minagawa AT, Schoeps FA, Montero RMJM. Aleitamento materno e níveis de hemoglobina em crianças menores de 2 anos em município do estado de São Paulo, Brasil. Rev Nutr. 2007; 20(2):149-57. doi: 10.1590/S1415-5273200 7000200004.

23. Souza SB, Szarfarc SC, Souza JM. Anemia no primeiro ano de vida em relação ao aleitamento materno. Rev Saúde Pública. 1997; 31(1):15-20.

24. Neuman NA, Tanaka OY, Szarfarc SC, Guimarães PR, Victora CG. Prevalência e fatores de risco para anemia no Sul do Brasil. Rev Saúde Pública. 2000; 34(1):56-63.

25. Silva LS, Giuglian ER, Aerts DR. Prevalência e determinantes de anemia em crianças de Porto Alegre, RS, Brasil. Rev Saúde Pública. 2001; 35(1):66-73.

26. Matta IEA, Veiga GV, Baião MR, Santos MMAS, Luiz RR. Anemia em crianças menores de cinco anos que freqüentam creches públicas do município do Rio de Janeiro, Brasil. Rev Saúde Materno Infantil. 2005; 5(3):349-57.

27. Chantry CJ, Howard CR, Auinger P. Full breastfeeding duration and risk for iron deficiency in U.S. infants. Breastfeed Med. 2007; 2(2):63-73.

28. Duncan B, Schifman RB, Corrigan JJ Jr, Schaefer C. Iron and the exclusively breast-fed infant from birth to six months. J Pediatr Gastroenterol Nutr. 1985; 4(3):421-5.

29. Pizarro F, Yip R, Dallman PR, Olivares M, Hertrampf E, Walter T. Iron status with different infant feeding regimens: relevance to screening and prevention of iron deficiency. J Pediatr. 1991; 118(5):687-92.

30. Calvo EB, Galindo AC, Aspres NB. Iron status in exclusively breast-fed infants. Pediatrics. 1992; 90(3):375-9.

31. Hopkins D, Emmett P, Steer C, Rogers I, Noble S, Emond A. Infant feeding in the second 6 months of life related to iron status: an observational study. Arch Dis Child. 2007; 92(10):850-4.

32. Souza SB, Szarfarc SC, Souza JMP. Prática alimentar no primeiro ano de vida, em crianças atendidas em centros de saúde escola do município de São Paulo. Rev Nutr. 1999; 12(2):167-74. doi: 10.1590/ S1415-5273199900020006.
33. Levy-Costa RB, Monteiro CA. Consumo de leite de vaca e anemia na infância no Município de São Paulo. Rev Saúde Pública. 2004; 38(6):797-803.

34. Gunnarsson BS, Thorsdottir I, Palsson G. Iron status in 2-years-old Icelandic children and associations with dietary intake and growth. Eur J Clin Nutr. 2004; 58(6):901-6.

35. Brasil. Ministério da Saúde. Dez passos para uma alimentação saudável: guia alimentar para crianças menores de dois anos. Brasília: Ministério da Saúde; 2010.

36. Shell-Duncan B, McDade T. Cultural and environmental barriers to adequate iron intake among northern Kenyan schoolchildren. Food Nutr Bull. 2005; 26(1):39-48.

37. Vitolo MR, Bortolini GA. Biodisponibilidade do ferro como fator de proteção contra anemia entre crianças de 12 a 16 meses. J Pediatr (Rio de Janeiro). 2007; 83(1):33-8.

38. World Health Organization. The prevalence of anaemia through primary health care: a guide for health administrators and programme managers. Geneva: WHO; 1989.

39. Engelmann MD, Sandström B, Michaelsen KF. Meat intake and iron status in late infancy: an intervention study. J Ped Gastroenterol Nutr. 1998; 26(1):26-33.

40. Hurrell RF. Bioavailability of iron. Eur J Clin Nutr. 1997; 51(Suppl 1):S4-8.

41. Cook JD, Reddy MB. Effect of ascorbic acid intake on nonheme-iron absorption from a complete diet. Am J Clin Nutr. 2001; 73(1):93-8.

42. García-Casal MN, Layrisse M, Solano L, Barón MA, Arguello F, Llovera D, et al. Vitamin A and $\beta$-Carotene can improve nonheme iron absorption from rice, wheat and corn by humans. J Nutr. 1998; 128(3): 646-50.

43. Hallberg L, Hoppe M, Andersson M, Hulthén L. The role of meat to improve the critical iron balance weaning. Pediatrics. 2003; 111(4 Pt 1):864-70.

44. Hallberg L, Rossander-Hultén L, Brune M, Gleerup A. Calcium and iron absorption: mechanism of action and nutritional importance. Eur J Clin Nutr. 1992; 46(5):317-27.

45. Kapur D, Sharma S, Agarwal KN. Effectiveness of nutrition education, iron supplementation or both on Iron Status in Children. Indian Pediatric. 2003; (40):1131-44.

46. Khoshnevisan F, Kimiagar M, Kalantaree N, Valaee $\mathrm{N}$, Shaheedee N. Effect of nutrition education and diet modification in iron depleted preschool children in nurseries in Tehran: a pilot study. Int J Vitam Nutr Res. 2004; 74(4):264-8. 
1062 | G.A. BORTOLINI \& M.R. VITOLO

47. World Health Organization. Infant and young child feeding counseling: an integrated course. Geneva: WHO; 2006.

48. Bassichetto KC, Rea MF. Aconselhamento em alimentação infantil: um estudo de intervenção. J Pediatr (Rio de Janeiro). 2008; 84(1):75-82.

49. Brasil. Ministério da Saúde. Estratégia nacional para alimentação complementar saudável. Brasil: Ministério da Saúde; 2010.

50. Hertrampf E, Olivares M, Walter T, Pizarro F. Anemia ferropriva en el lactante: erradicación con leche fortificada con hierro. Rev Méd Chile. 1990; 118: 1330-7.

51. Torres MA, Sato K, Lobo NF, Souza Queiroz S. Efeito do uso de leite fortificado com ferro e vitamina C sobre os níveis de hemoglobina e condição nutricional de crianças menores de 2 anos. Rev Saúde Pública. 1995; 29(4):301-7.

52. Vitolo MR, Aguirre NC, Kondo MR, Giuliano Y, Ferreira N, Lopez FA. Impacto do uso de cereal adicionado de ferro sobre os níveis de hemoglobina e a antropometria de pré-escolares. Rev Nutr. 1998; 11(2):163-71. doi: 10.1590/S1415-52731998000 200007.

53. Brasil. Ministério da Saúde. RDC 344, 13 dezembro de 2002. Regulamento técnico para fortificação das farinhas de trigo e das farinhas de milho com ferro e ácido fólico. Diário Oficial da União. 1998 31 dez; Seção 1.

54. Assunção MC, Santos IS, Barros AJ, Gigante DP, Victora CG. Efeito da fortificação de farinhas com ferro sobre anemia em pré-escolares, Pelotas, RS. Rev Saúde Pública. 2007; 41(4):539-48.

55. Sherry B, Mei Z, Yip R. Continuation of the decline in prevalence of anemia in low-income infants and childrens in five states. Pediatrics. 2001; 107(4): 677-82.

56. Looker AC, Dallman PR, Carroll MD, Gunter EW, Johnson CL. Prevalence of iron deficiency in the United States. Prevalence of iron deficiency in the United States. J Am Med Assoc. 1997; 277(12): 973-6.

57. Pizarro F. Fortificación de alimentos com hierro: la experiência chilena. Rev Méd Minas Gerais. 2007; 17:S80-S5.
58. Hercberg S, Preziosi P, Galan P. Iron deficiency in Europe. Public Health Nutr. 2001; 4(2):537-545.

59. Herrera MP. Estrategias y acciones para combatir la anemia y la deficiencia de hierro: la experiencia de Cuba en la fortificación de alimentos con hierro. Rev Méd Minas Gerais. 2007; 17:S86-S9.

60. Fontes F. Politicas de combate de carencias de micronutrientes em Panamá. Rev Méd de Minas Gerais. 2007; 17:S74-S9.

61. Sprinkles Global Health initiative: about sprinkles. [cited 2009 May]. Available from: <http://www. sghi.org/index.html>.

62. Sociedade Brasileira de Pediatria. Manual de orientação: departamento de nutrologia. São Paulo: Sociedade Brasileira de Pediatria; 2006.

63. Allen LH. Iron supplements: scientific issues concerning efficacy and implications for research and programs. J Nutr. 2002; 132:813s-819s.

64. Brasil. Ministério da Saúde. Manual operacional: programa nacional de suplementação de ferro. Brasília; 2005. Disponível em: <www.saude. gov.br/ alimentacao>.

65. Engstrom EM. Efetividade da suplementação diária e semanal com sulfato ferroso na prevenção da anemia em crianças menores de 1 ano de idade: ensaio de campo randomizado por grupos [dissertação]. Rio de Janeiro: Fiocruz; 2006.

66. Bortolini GA, Vitolo MR. Baixa adesão à suplementação de ferro entre lactentes usuários de serviço público de saúde. Pediatria (São Paulo). 2007; 29(3):176-82.

67. Andres NC. Disorders of iron metabolism. New Engl J Med. 1999; 341(26):1986-95.

68. Galloway R, McGuire J. Determinants of compliance with iron supplementation:supplies, side effects, or psychology? Social Sci Med. 1994; 39:381-90.

69. Vitolo MR, Boscaini C, Bortolini GA. Baixa escolaridade como fator limitante para o combate à anemia entre gestantes. Rev Bras Ginecol Obstr. 2006; 28(6):331-9.

Recebido em: 16/5/2008

Versão final reapresentada em: 17/6/2010 Aprovado em: 22/9/2010 